03

\title{
Об устойчивости сильно заряженной капли, подвешенной в суперпозиции гравитационного и электростатического полей
}

\author{
() С.О. Ширяева, Н.А. Петрушов, А.И. Григорьев \\ Ярославский государственный университет им. П.Г. Демидова, \\ 150000 Ярославль, Россия \\ e-mail: shir@uniyar.ac.ru
}

Поступило в Редакцию 23 ноября 2017 г.

В окончательной редакции 18 февраля 2019 г.

Принято к публикации 25 февраля 2019 г.

Для сильно заряженной капли, подвешенной в неподвижном состоянии в суперпозиции гравитационного и электростатического полей, найдены критические условия реализации ее неустойчивости по отношению к собственному и индуцированному зарядам. Все расчеты проведены в четвертом порядке малости по величине стационарной деформации сферической формы капли и первом порядке по безразмерной амплитуде ее капиллярных осцилляций. Найдены зависимости величин критических параметров Рэлея $W_{c r}$ и Тейлора $w_{c r}$ от радиуса изначально сферической капли, плотности, коэффициента поверхностного натяжения, ускорения свободного падения и от номера моды осцилляций, отличные от таковых для свободной капли. С увеличением номера моды критическое значение параметра Рэлея растет и выходит на асимптотику $W_{c r} \approx 0.95$, а критическое значение параметра $w_{c r}$ снижается и выходит на асимптотику $w_{c r} \approx 1 / 25 \cdot 10^{-4}$. Указанные изменения $W_{c r}$ и $w_{c r}$ объясняются наличием условия неподвижности центра масс в подвесе, связывающего $W_{c r}, w_{c r}$ и ускорение поля сил тяжести.

Ключевые слова: заряженная капля, электростатический подвес, осцилляции.

\section{Введение}

Исследование устойчивости заряженной капли во внешних силовых полях представляет интерес в связи с многочисленными академическими, техническими и технологическими приложениями, в частности, в связи с проблемой проверки справедливости критерия Рэлея [1], который в конце позапрошлого века теоретически вывел критерий устойчивости по отношению к собственному заряду сферической капли электропроводной несжимаемой жидкости в вакууме. C тех пор и до наших дней неоднократно предпринимаются попытки экспериментальной проверки этого критерия (см., например, [2-10]). Чтобы экспериментально его проверить, необходимо сначала подвесить сильно заряженную (почти до рэлеевского предела) каплю в каком-либо бесконтактном подвесе [2-10]. А потому для ее удержания во взвешенном состоянии в подвесах используются различные силовые поля, деформирующие каплю и, следовательно, искажающие результаты измерения критерия неустойчивости [11].

Использование бесконтактных подвесов связано также с современными технологиями получения высокочистых веществ и определения физико-химических характеристик чистых жидкостей.

В связи со сказанным, будем исследовать устойчивость осцилляций сильно заряженной капли, подвешенной в неподвижном состоянии в суперпозиции гравитационного и слабого электростатического полей.

\section{Постановка задачи}

Пусть имеется капля несжимаемой идеально проводящей жидкости, несущая заряд $Q$, подвешенная в электростатическом подвесе, когда коллинеарные внешнее однородное электростатическое поле напряженностью $\mathbf{E}_{0}$ и гравитационное поле $\mathbf{g}$ удерживают ее в висячем положении. Примем, что жидкость имеет плотность $\rho$ и коэффициент поверхностного натяжения $\sigma$. В отсутствие электростатического и гравитационного полей капля имеет сферическую форму с радиусом $R$.

Будем решать задачу о расчете осцилляций выше описанной капли в сферической системе координат с началом в центре масс капли. Все решение будем проводить в безразмерных переменных, в которых $\rho=\sigma=R=1$.

Проведем расчет осцилляций сильно заряженной капли в слабом электростатическом поле, коллинеарном гравитационному полю, когда капля имеет равновесную форму, близкую к вытянутому вдоль электростатического поля сфероиду $[12,13]$, свободная поверхность которого описывается выражением [14]

$$
\begin{gathered}
r(\theta)=1+h(\theta) \equiv 1+a_{0}^{(4)} P_{0}(\mu)+\left(a_{2}^{(2)}+a_{2}^{(4)}\right) P_{2}(\mu) \\
+a_{3}^{3} P_{3}(\mu)+a_{4}^{(4)} P_{4}(\mu) \\
\mu \equiv \cos \theta
\end{gathered}
$$

где $P_{n}(\mu)$ - полиномы Лежандра, $h(\theta)$ - равновесное отклонение во внешних электростатическом и гравитационном полях формы капли от сферической, коэффициенты $a_{n}^{(m)}$ определяют вклад различных полиномов 
Лежандра в формирование равновесной формы капли. Верхний индекс $m$ в $a_{n}^{(m)}$ указывает на порядок малости, в котором он рассчитывался, по величине стационарной деформации исходной сферической формы капли. Коэффициенты $a_{n}^{(m)}$ достаточно громоздки, не имеют прямого отношения к теме работы и приведены в [14]. Форма капли, описываемая выражением (1), с хорошей точностью может считаться вытянутой сфероидальной. Отклонение от сфероидальности обусловлено наличием слагаемого $a_{3}^{3} P_{3}(\mu)$ в (1), которое имеет величину порядка нескольких процентов от общей величины $|h(\theta)|$.

Математическая формулировка задачи имеет вид

$$
\begin{gathered}
\partial_{t} \mathbf{u}(\mathbf{r}, t)+(\mathbf{u}(\mathbf{r}, t), \nabla) \mathbf{u}(\mathbf{r}, t)=-\nabla p_{i n}(\mathbf{r}, t)+\mathbf{g} ; \\
\operatorname{div} \mathbf{u}(\mathbf{r}, t)=0 ; \quad \operatorname{div} \mathbf{E}(\mathbf{r}, t)=0 ; \\
r \rightarrow \infty ; \quad \mathbf{E}(\mathbf{r}, t) \rightarrow \mathbf{E}_{0} ; \quad r \rightarrow 0 ; \quad \mathbf{u}(\mathbf{r}, t) \rightarrow 0 ; \\
r=1+h(\theta)+\xi(\theta, t): \\
\frac{d F(\mathbf{r}, t)}{d t}=0 ; \quad d F(\mathbf{r}, t) \equiv r-1-h(\theta)-\xi(\theta, t) ; \\
\Phi(\mathbf{r}, t)=\Phi_{s} ; \\
p_{E}(\mathbf{r}, t)+p_{i n}(\mathbf{r}, t)-p_{0}-p_{\sigma}(\mathbf{r}, t)=0 ; \\
p_{E}(\mathbf{r}, t) \equiv \frac{E^{2}(\mathbf{r}, t)}{8 \pi} ; \quad p_{\sigma} \equiv \operatorname{div} \mathbf{n}(\mathbf{r}, t) ; \\
\mathbf{n}(\mathbf{r}, t) \equiv \frac{\nabla F(\mathbf{r}, t)}{|\nabla F(\mathbf{r}, t)|} .
\end{gathered}
$$

Здесь $\mathbf{u}(\mathbf{r}, t)$ и $p_{\text {in }}(\mathbf{r}, t)-$ поля скоростей и гидродинамического давления, $\mathbf{E}(\mathbf{r}, t)$ - напряженность электрического поля в окрестности капли, $\Phi_{s}-$ постоянный электростатический потенциал поверхности капли, $\xi(\theta, t)$ - возмущение равновесной ее формы, обязанное своим происхождением тепловому движению молекул жидкости [15].

Модуль обезразмеренного на $R$ возмущения $\xi(\theta, t)$ примем в качестве первого малого параметра $\varepsilon$, а модуль обезразмеренной напряженности поля $E$ в качестве второго малого параметра $\eta$.

Условия сохранения объема, заряда и неподвижности центра масс запишутся в виде

$$
\begin{gathered}
\iiint_{V}=\frac{4}{3} \pi, \\
V \equiv\{0 \leq r \leq 1+h(\theta)+\xi(\theta, t) ; 0 \leq \theta \leq \pi ; 0 \leq \varphi \leq 2 \pi\} \\
\iint_{S}(\mathbf{n}(\mathbf{r}, t), \mathbf{E}(\mathbf{r}, t)) d S=4 \pi Q, \\
S \equiv\{r=1+h(\theta)+\xi(\theta, t) ; 0 \leq \theta \leq \pi ; 0 \leq \varphi \leq 2 \pi\}, \\
\iiint_{V} \mathbf{r} d V=0 .
\end{gathered}
$$

\section{Расчет осцилляций поверхности капли}

В задаче (2) с граничными (3) и дополнительными (4) условиями перейдем от поля скоростей в капле к полю гидродинамического потенциала $\psi(r, \theta, t)$ и от поля напряженности к полю электростатического потенциала $\Phi(r, \theta, t)$ на основе соотношений $[16,17]$

$$
\mathbf{u}(r, \theta, t)=\nabla \psi(r, \theta, t) ; \quad \mathbf{E}(r, \theta, t)=-\nabla \Phi(r, \theta, t) .
$$

Задачу будем решать асимптотическим методом путем разложения по малым параметрам [18]: в первом порядке малости по $\varepsilon$ и в четвертом порядке по $\eta$. Так как движение внутри капли обусловлено наличием возмущения на ее поверхности, примем $|\psi| \propto|\xi| \sim \varepsilon$. Полученные решения будем выражать через параметры Рэлея $W \equiv \frac{Q^{2}}{16 \pi \sigma R^{3}}[1]$ и Тейлора $w \equiv \frac{E_{0}^{2} R}{16 \pi \sigma}[19]$, характеризующие устойчивость капли по отношению к собственному и индуцированному зарядам соответственно:

$$
w \sim E_{0}^{2} \sim \eta^{2} ; \quad W \sim Q^{2} \sim \eta^{0} .
$$

Следует отметить, что в принятых безразмерных переменных параметры Рэлея и Тейлора имеют вид $W \equiv \frac{Q^{2}}{16 \pi}$ и $w \equiv \frac{E_{0}^{2}}{16 \pi}$. Из такой записи параметра Тейлора можно оценить величину второго малого параметра $\eta$, задаваясь величинами $w \sim 10^{-4}$ (характерной для электростатического подвеса при $R \sim 10^{-2} \mathrm{~cm}$ ) и $\eta \sim 0.07$.

Электростатический потенциал представим в виде разложения

$$
\Phi(r, \theta, t)=\Phi^{(0)}(r, \theta)+\varepsilon \Phi^{(1)}(r, \theta, t)+O\left(\varepsilon^{2}\right),
$$

где $\Phi^{(0)}(r, \theta)$ - электростатический потенциал в окрестности капли равновесной формы $(1), \Phi^{(1)}(r, \theta, t)-$ поправка к равновесному электростатическому потенциалу $\Phi^{(0)}(r, \theta)$, возникающая в результате теплового капиллярно-волнового [15] возмущения поверхности капли. Разобьем задачу (1)-(4) с учетом (5), (6) на нулевой и первый порядки малости по $\varepsilon$.

Задача нулевого порядка малости описывает невозмущенную (равновесную) поверхность капли и определяет электростатическое поле в ее окрестности, когда поверхность описывается выражением (1):

$$
\begin{gathered}
\Delta \Phi^{(0)}(r, \theta)=0 ; \quad r \rightarrow \infty: \quad-\nabla \Phi^{(0)}(r, \theta) \rightarrow E_{0} \mathbf{e}_{z} ; \\
r=1+h(\theta): \quad \Phi^{(0)}(r, \theta)=\Phi_{s}, \\
\iint_{S}\left(\mathbf{n}(\mathbf{r}, t), \nabla \Phi^{(0)}(r, \theta)\right) d S=-4 \pi Q, \\
S \equiv\{r=1+h(\theta) ; 0 \leq \theta \leq \pi ; 0 \leq \varphi \leq 2 \pi\} .
\end{gathered}
$$

где $\mathbf{e}_{z}-$ орт оси $O Z$, совпадающий по направлению с $\mathbf{E}_{0}$. 
Решая выписанную задачу, можно показать [14], что потенциал электростатического поля в окрестности капли с точностью до слагаемых $\sim \eta^{4}$ будет иметь вид

$$
\begin{aligned}
\Phi^{(0)}(r, \theta)= & \frac{4 \sqrt{\pi W}}{r}+\sqrt{w} A_{1}+w A_{2}+\sqrt{w^{3}} A_{3} \\
& +w^{2} A_{4}+O\left(\eta^{5}\right) ;
\end{aligned}
$$

где коэффициенты $A_{j}$ не приведены из-за громоздкости. Их можно найти в [14].

Задача первого порядка малости, определяющая собственно осцилляции капли, запишется в виде

$$
\begin{gathered}
\Delta \Phi^{(1)}(r, \theta, t)=0 ; \quad \Delta \psi(r, \theta, t)=0 ; \\
r \rightarrow \infty: \quad \Phi^{(1)}(r, \theta, t) \rightarrow 0 ; \quad r \rightarrow 0: \quad \psi(r, \theta, t) \rightarrow 0 \\
r=1+h(\theta): \quad-\partial_{t} \xi(\theta, t)-\frac{\partial_{\theta} h(\theta) \partial_{\theta} \psi(r, \theta, t)}{r^{2}} \\
+\partial_{r} \psi(r, \theta, t)=0, \\
\Phi^{(1)}(r, \theta, t)+\xi(\theta, t) \partial_{r} \Phi^{(0)}(r, \theta)=0, \\
p_{i n}^{(1)}(r, \theta, t)+p_{E Q}^{(1)}(\theta, t)+p_{g}^{(1)}(\theta, t)-p_{\sigma}^{(1)}(\theta, t)=0, \quad(10) \\
p_{i n}^{(1)}(r, \theta, t)=f_{1}(t)-\partial_{t} \psi(r, \theta, t)-g(\xi(0, t)-\xi(\theta, t) \cos \theta), \\
\times \partial_{r} \Phi^{(1)}(r, \theta, t)+\frac{\xi(\theta, t)}{4 \pi r^{2}}\left(-\left(\Phi^{(0)}(r, \theta)\right)^{2}+r \partial_{\theta} \Phi^{(0)}(r, \theta)\right. \\
\left.\times \partial_{r, \theta} \Phi^{(0)}(r, \theta)+r^{3} \partial_{r} \Phi^{(0)}(r, \theta) \partial_{r, r} \Phi^{(0)}(r, \theta)\right) \\
p_{\sigma}^{(1)}(\theta, t)=\frac{1}{r^{2} \sqrt{r^{2}+\left(\partial_{\theta} h(\theta)\right)^{2}}}\left[\left(2 r^{5}+5 r^{3}\left(\partial_{\theta} h(\theta)\right)^{2}\right.\right. \\
+2 r^{4} \partial_{\theta \theta} h(\theta)-r^{2}\left(\partial_{\theta} h(\theta)\right)^{2} \partial_{\theta \theta} h(\theta)+\left(2 r^{4} \partial_{\theta} h(\theta)\right. \\
\left.\left.+3 r^{2}\left(\partial_{\theta} h(\theta)\right)^{3}+\left(\partial_{\theta} h(\theta)\right)^{5}\right) \operatorname{ctg} \theta\right) \xi(\theta, t)+r^{3} \\
\times\left(r^{2}+\left(\partial_{\theta} h(\theta)\right)^{2}\right) \partial_{\theta \theta} \xi(\theta, t)+\left(r^{3}\left(r^{2}+\left(\partial_{\theta} h(\theta)\right)^{2}\right) \operatorname{ctg} \theta\right. \\
\left.\left.-3 r^{2}\left(\left(\partial_{\theta} h(\theta)\right)^{2}+r \partial_{\theta \theta} h(\theta)\right) \partial_{\theta} h(\theta)\right) \partial_{\theta} \xi(\theta, t)\right]=0 .
\end{gathered}
$$

Равенство нулю граничных условий (8), (9) можно обеспечить, положив равными нулю коэффициенты при каждом из полиномов Лежандра в силу их ортогональности. Сохраним в коэффициентах слагаемые, имеющие величину $\sim \eta^{4}$ включительно. Амплитуды $A_{n}(t)$ и $B_{n}(t)$ гидродинамического и электрического потенциалов также представим в виде разложений по малому параметpy $\eta$ :

$$
\begin{aligned}
& A_{n}(t)=\sum_{i=0}^{4} \eta^{i} A_{n}^{(i)}(t)+O\left(\eta^{5}\right) \\
& B_{n}(t)=\sum_{i=0}^{4} \eta^{i} B_{n}^{(i)}(t)+O\left(\eta^{5}\right) .
\end{aligned}
$$

В итоге, выражая с помощью (8), (9) амплитудные коэффициенты $A_{n}^{(i)}(t)$ и $A_{n}^{(i)}(t)$ через $\alpha_{n}(t)$, из динамического граничного условия (10) получим эволюционное уравнение для $\alpha_{n}(t)$, которое имеет вид (см. [14])

$$
\begin{aligned}
& \frac{\partial^{2} \alpha_{n}(t)}{\partial t^{2}}+\omega_{n}^{2} \alpha_{n}(t)=\eta N_{n \pm 1}^{(1)} \alpha_{n \pm 1}(t)+\eta^{2}\left(N_{n \pm 2}^{(2)} \alpha_{n \pm 2}(t)\right. \\
& \left.+N_{n \pm 2}^{(3)} \frac{\partial^{2} \alpha_{n \pm 2}(t)}{\partial t^{2}}\right)+\eta^{3}\left(N_{n \pm 1}^{(4)} \alpha_{n \pm 1}(t)+N_{n \pm 3}^{(5)} \alpha_{n \pm 3}(t)\right. \\
& \left.+N_{n \pm 1}^{(6)} \frac{\partial^{2} \alpha_{n \pm 1}(t)}{\partial t^{2}}+N_{n \pm 3}^{(7)} \frac{\partial^{2} \alpha_{n \pm 3}(t)}{\partial t^{2}}\right)+\eta^{4}\left(N_{n \pm 2}^{(8)} \alpha_{n \pm 2}(t)\right. \\
& \left.+N_{n \pm 4}^{(9)} \alpha_{n \pm 4}(t)+N_{n \pm 2}^{(10)} \frac{\partial^{2} \alpha_{n \pm 2}(t)}{\partial t^{2}}+N_{n \pm 4}^{(11)} \frac{\partial^{2} \alpha_{n \pm 4}(t)}{\partial t^{2}}\right) .
\end{aligned}
$$

$N_{n}^{(i)}$ - громоздкие коэффициенты, зависящие от физикохимических параметров задачи, здесь не приводятся; $\omega_{n}-$ частота, в общем случае комплексная:

$$
\begin{gathered}
\omega_{n}^{2}=\omega_{n, 0}^{2}+\eta^{2} \delta \omega_{n}^{2}, \\
\omega_{n, 0}^{2}=n(n-1)(2+n-4 W), \\
\delta \omega_{n}^{2} \equiv \omega_{n, 1}^{2}+\eta^{2} \omega_{n, 2}^{2},
\end{gathered}
$$




$$
\begin{gathered}
\omega_{n, 1}^{2}=\frac{9 n w}{(3+2 n)(2 n+1)(2 n-1)(1-W)}\left(2-4 W+n(7-8 W)+n^{2}(17-8 W)-3 n^{3}(7-8 W)-n^{4}(23-16 W)-2 n^{5}\right) \\
\omega_{n, 2}^{2}=\frac{9 n w^{2} \cdot H(W, n)}{70(2 n-1)^{2}(2 n+1)^{2}(2 n-3)(2 n+3)^{2}(2 n+5)(1-W)^{2}(3-2 W)(4 W-5)}
\end{gathered}
$$

$H(W, n)$ - весьма громоздкий коэффициент, зависящий только от $W$ и $n$. Следует отметить, что выражение (16) получено в асимптотической процедуре по двум малым параметрам $\varepsilon$ и $\eta$ [18], что означает малость добавки $\delta \omega_{n}^{2}$ по порядку величины по сравнению с $\omega_{n, 0}^{2}$.

Как видно из эволюционного уравнения (15), в пределах рассматриваемой нами точности по малым параметрам $\varepsilon$ и $\eta$, вместе с модой, возбужденной изначально, на поверхности капли будут возбуждаться еще восемь связанных с ней мод [14].

Решения эволюционного уравнения находятся методом последовательных приближений с точностью $\sim \eta^{4}$ в виде суперпозиции косинусов (со сложными коэффициентами), зависящих от частот связанных мод (см. [14]).

\section{Неустойчивость осцилляций заряженной капли \\ в электростатическом подвесе}

Реализация неустойчивости осцилляций капли соответствует условиям, при которых квадрат ее частоты принимает отрицательные значения, тем самым критические условия определяются обращением в нуль квадрата частоты: $\omega_{n}^{2}=0$. Используя (16), получим уравнение, связывающее критические значения параметров Рэлея и Тейлора:

$$
\omega_{n, 0}^{2}+\eta^{2} \delta \omega_{n}^{2}=0
$$

В выражении квадрата частоты $\omega_{n}^{2}(16)$ нулевой порядок малости по $\eta$ является квадратом частоты осцилляций сферической заряженной капли (17) в отсутствие электростатического и гравитационного полей. Их наличие приводит к появлению поправки $\delta \omega_{n}^{2}$ к частоте осцилляций (17), приводящей к смещению условий реализации неустойчивости.

Расчеты по (19) с учетом (17), (18) показывают, что расчетное значение параметра Рэлея $W$ для капли с учетом неподвижности ее центра масс (в суперпозиции гравитационного и электростатического полей) меньше полученного Рэлеем для сферической капли $\left(W_{c r}=1\right)[1,20]$ и составляет $W_{c r} \approx 0.977$, что можно объяснить наличием стационарной деформации сферической формы.

Равновесная форма сильно заряженной капли в суперпозиции гравитационного и однородного электростатического полей не сферична. Расчеты показывают, что она с высокой степенью точности (см. формулу (1)) может быть аппроксимирована вытянутым вдоль электростатического поля сфероидом. Причем такая форма капли обеспечивается не внешним электростатическим полем, которое может быть весьма слабым (только чтобы обеспечить подвешивание капли и, фактически, просто задает выделенное направление), а близким по величине к критическому значению собственным зарядом капли.

Все расчеты (здесь и ниже при построении графиков) проводились при $n=2$ для капли жидкости плотностью $\rho=1 \mathrm{~g} / \mathrm{cm}^{2}$, радиусом $R=0.01$, с коэффициентом поверхностного натяжения $\sigma=73 \mathrm{dyn} / \mathrm{cm}$ в поле сил тяжести $g=980.7 \mathrm{~cm} / \mathrm{s}^{2}$.

На рис. 1, $a$ представлена рассчитанная (по (19)) зависимость критического параметра Рэлея $W_{c r}$ от номера моды капли в условиях электростатического подвеса. Как видно из рисунка, с увеличением номера моды критическое значение параметра Рэлея увеличивается. Причем с увеличением номера моды критическое значение параметра Рэлея выходит на асимптотику $W_{c r} \approx 0.95$. Напомним, что критическое по Рэлею значение $W$ для заряженной капли в отсутствие внешних силовых полей равно единице.

На рис. $1, b$ представлена зависимость критического параметра Тейлора $w_{c r}$ от номера моды осцилляций сильно заряженной капли при тех же условиях. Ситуация аналогична (с точностью до наоборот), приведенной на рис. 1, $a$ : критическое значение параметра $w_{c r}$ снижается с увеличением номера моды и выходит на асимптотику $w_{c r}=1.25 \cdot 10^{-4}$. Это объясняется наличием условия неподвижности центра масс: $g=12 \sqrt{w W}$, так что три параметра $W_{c r}, w_{c r}$ и $g$ оказываются не независимыми, а связанными. Критическое для незаряженной капли во внешнем электростатическом поле значение параметра Тейлора $w_{c r} \approx 0.05$.

На рис. 2-5 приведены зависимости $W_{c r}$ и $w_{c r}$ от физических параметров задачи: $R, \rho, \sigma, g$, взятых в размерном виде. Для свободной сферической капли указанные зависимости определяются аналитическими выражениями:

$$
W_{c r} \equiv \frac{Q^{2}}{16 \pi \sigma R^{3}}=1 ; \quad w_{c r} \equiv \frac{E^{2} \sigma}{16 \pi R}=1
$$

В частности, зависимостей от $\rho$ и $g$ в (20) нет совсем. Но при рассмотрении устойчивости заряженной капли в электростатическом подвесе такие зависимости появляются.

На рис. 2, $а$ приведена зависимость критического параметра Рэлея $W_{c r}$ от ее радиуса. Видно, что с уменьшением радиуса капли критическое значение параметра Рэлея увеличивается и стремится к $W_{c r}=1$. 

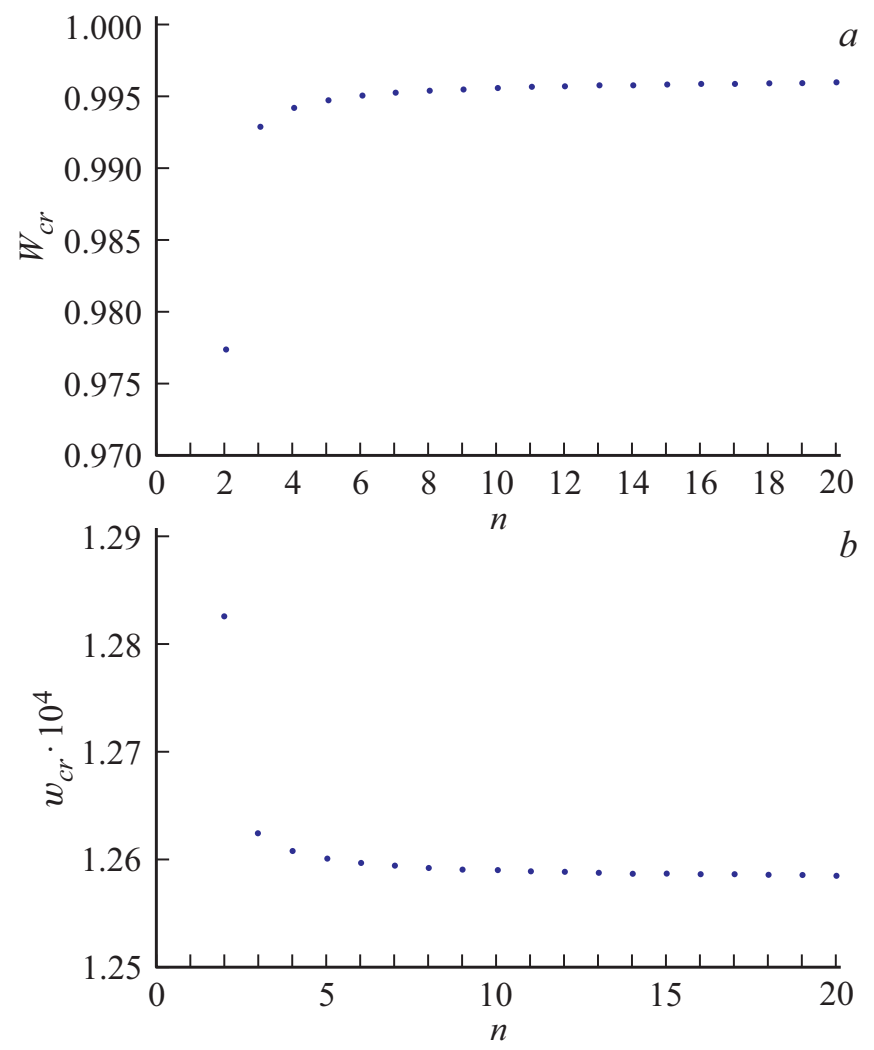

Рис. 1. $a-$ зависимость критического значения параметра Рэлея $W_{c r}$ от номера моды заряженной капли, неподвижной в электростатическом подвесе; $b-$ зависимость критического значения параметра Тейлора $w_{c r}$ от номера моды.

На рис. 2, $b$ приведена зависимость критического значения параметра Тейлора $w_{c r}$ капли от радиуса капли. Из рис. 2 видно, что зависимости $W_{c r}$ и $w_{c r}$ от $R$ противоположны, но не такие, какие следовали бы из (20).

Зависимость критического параметра Рэлея $W_{c r}$ капли от величины плотности жидкости $\rho$ практически линейна, немного выпукла. Параметр $W_{c r}$ убывает от 1 до 0.92 с увеличением $\rho$ от нуля до $2.7 \mathrm{~g} / \mathrm{cm}^{3}$. На рис. 3 представлена зависимость критического параметра Тейлора $w_{c r}$ капли от величины плотности жидкости. Видно, что с ростом величины $\rho$, критическое значение параметpa $w_{c r}$ увеличивается примерно по параболическому закону. Для свободной изначально сферической капли зависимости от $\rho$ ни $W_{c r}$, ни $w_{c r}$ быть не должно.

На рис. 4, $a$ представлена зависимость критического параметра Рэлея $W_{c r}$ капли от величины коэффициента поверхностного натяжения. Видно, что с ростом величины коэффициента поверхностного натяжения критическое значение параметра Рэлея для капли в подвесе увеличивается по выпуклой кривой, а не линейно, как это следует из (20) для свободной капли.

Противоположная зависимость для параметра Тейлоpa $w_{c r}$ для повешенной капли на рис. $4, b$ свидетельствует о снижении $w_{c r}$ с ростом $\sigma$, тогда как, согласно (20), для свободной капли должен наблюдаться линейный рост $w_{c r}$ с ростом $\sigma$.

Расчеты показывают, что зависимость критического значения параметра Рэлея $W_{c r}$ капли в электростатическом подвесе от величины ускорения свободного падения $g$ практически линейна. Параметр $W_{c r}$ убывает от 1 до $\approx 0.97$ с увеличением $g$ от нуля до $1022 \mathrm{~cm} / \mathrm{g}^{2}$. А вот расчетная по (19) зависимость критического параметра Тейлора $w_{c r}$ от $g$ более сложна (рис. 5). Видно, что с увеличением $g$ параметр $w_{c r}$ растет по примерно параболическому закону.
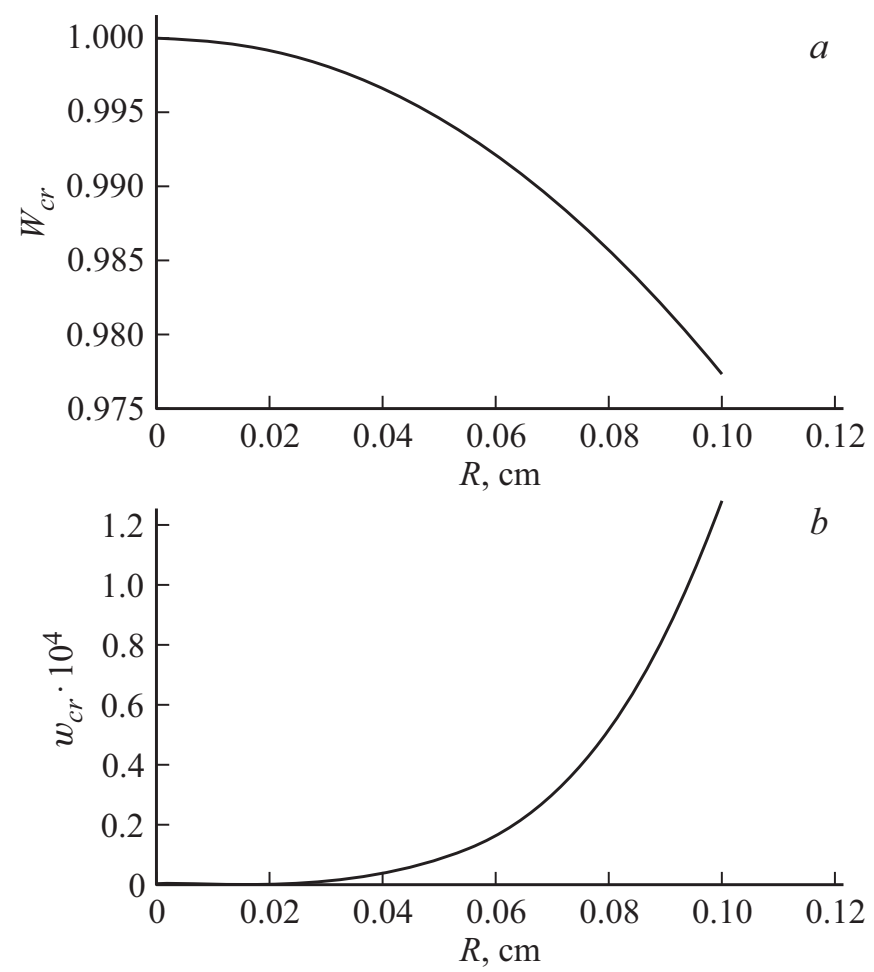

Рис. 2. $a-$ зависимость критического значения параметра Рэлея $W_{c r}$ от радиуса исходной капли; $b-$ зависимость критического значения параметра Тейлора $w_{c r}$ капли от радиуса исходной капли при аналогичных условиях.

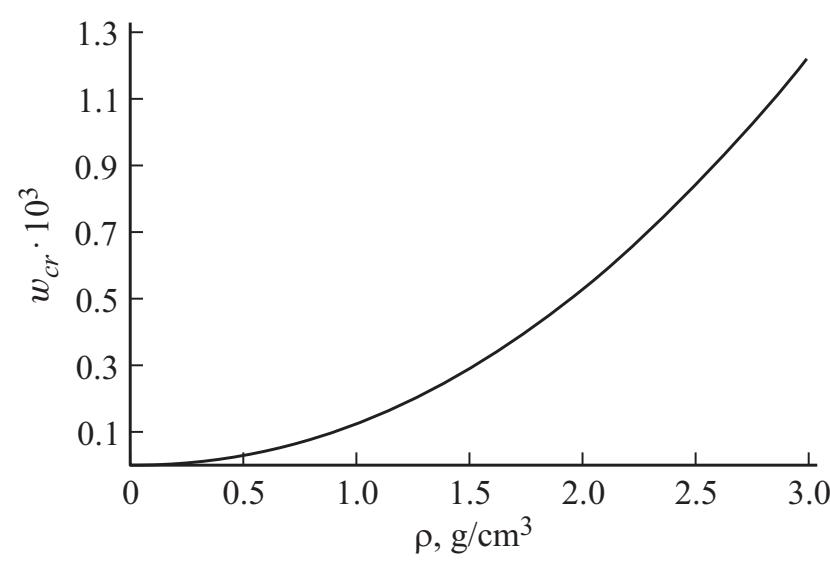

Рис. 3. Зависимость критического параметра Тейлора $w_{c r}$ капли от плотности жидкости. 

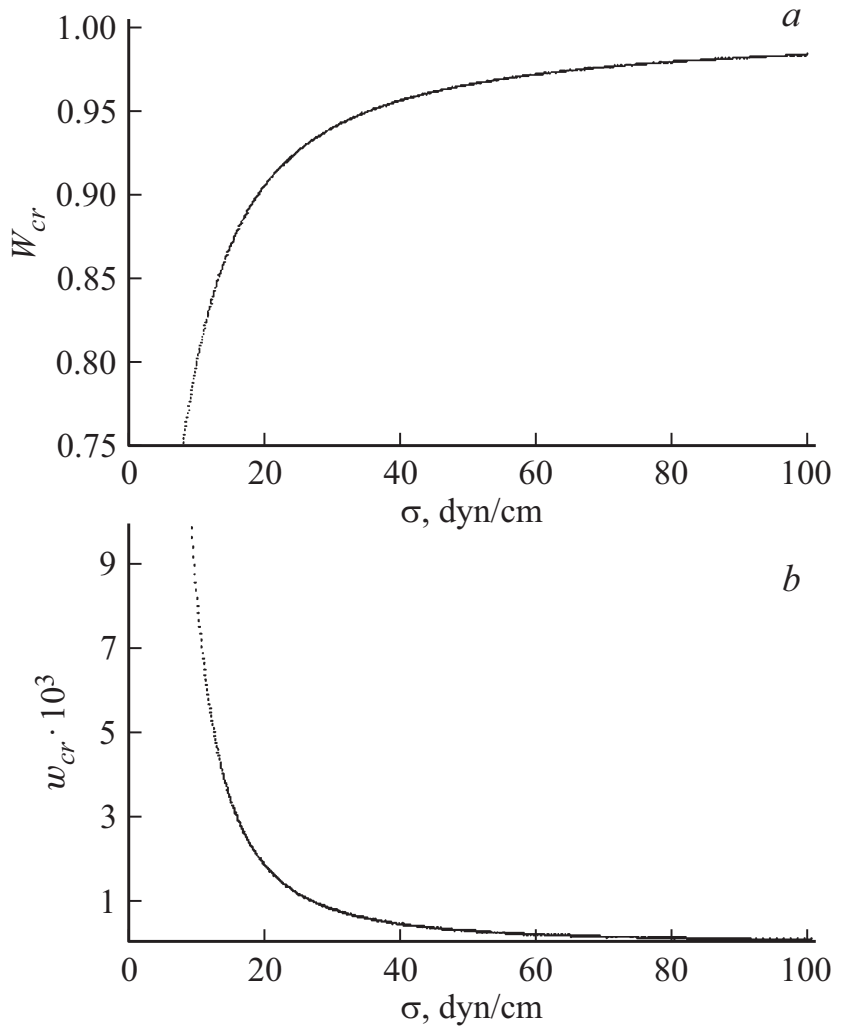

Pис. 4. $a-$ зависимость критического параметра Рэлея $W_{c r}$ капли от величины коэффициента поверхностного натяжения жидкости капли; $b-$ зависимость критического параметра Тейлора $w_{c r}$ капли от величины коэффициента поверхностного натяжения жидкости капли.

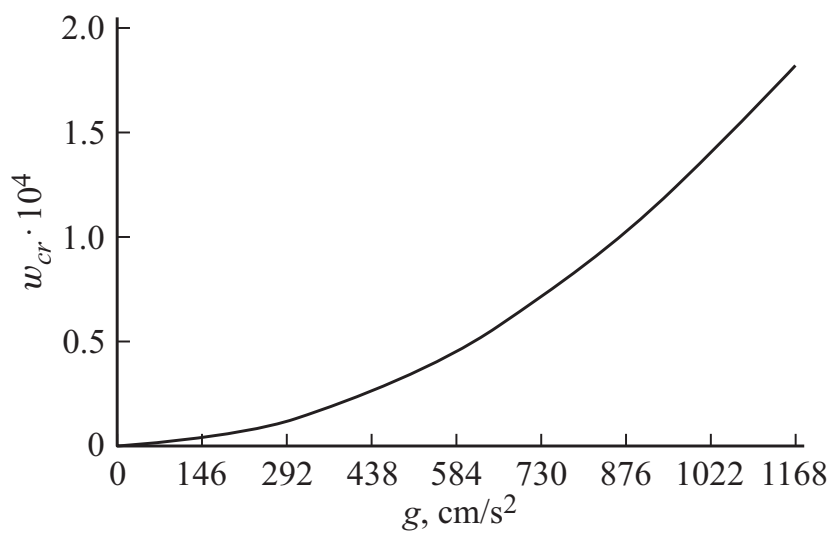

Рис. 5. Зависимость критического параметра Тейлора $w_{c r}$ капли от величины ускорения свободного падения.

Сказанное выше означает, что закономерности реализации неустойчивости свободной электропроводной заряженной капли и капли в электростатическом подвесе несколько различаются. Свободная капля изначально сферична, и ее неустойчивость начинается с неустойчивости основной моды, когда капля вытягивается в сфероид; поверхностный заряд, достигший критической величины на сферической капле, становится закритиче- ским на вершинах сфероида. При этом возбуждаются более высокие, чем основная, моды, и капля сбрасывает избыточный заряд путем эмиссии высокодисперсных сильно заряженных мелких капелек. Отметим, что напряженность электрического поля у поверхности капли $E$ связана с поверхностной плотностью заряда $\chi$ простым соотношением

$$
E \equiv 4 \pi \chi
$$

Для заряженной капли, висящей в электростатическом подвесе, равновесная форма капли уже сфероидальна [13], причем величина экцентриситета сфероида конечна. При приближении к критическому состоянию эксцентриситет сфероидальной капли увеличивается, а вместе с ним увеличивается и поверхностная плотность заряда $\chi$ на вершинах. При потере устойчивости основной модой ее амплитуда начинает неограниченно расти, а вместе с ней станет увеличиваться и эксцентриситет сфероидальной капли, генерируя на вершинах сфероида неустойчивость более высоких мод и выброс дочерних капелек, как и отмечалось в экспериментах [2-9]. Отметим, что, согласно проведенным в настоящей работе расчетам, разница в критических условиях реализации неустойчивости высоких мод уменьшается с ростом номера моды до весьма малой величины.

В конце проведенных рассуждений следует отметить, что равновесная форма капли в подвесе рассчитывалась в приближении $\sim \eta^{4}$, а амплитуда тепловых осцилляций $\sim \varepsilon$. Это обстоятельство приводит к соотношению между использованными малыми параметрами: $\eta^{5} \sim \varepsilon$. Расчеты корректны только при выполнении указанного условия.

\section{Заключение}

Найдены критические зависимости зарядового (Рэлея) и полевого (Тейлора) параметров, при которых реализуется неустойчивость заряженной капли, неподвижной в суперпозиции гравитационного и электростатического полей, от номера моды осцилляций, от радиуса исходной сферической формы капли, плотности жидкости, величины ее коэффициента поверхностного натяжения и ускорения свободного падения, отличные от аналогичных зависимостей свободной сферической капли. Обнаружено, что критические значения параметров Релея и Тейлора для заряженной капли, подвешенной во внешних гравитационном и электростатическом полях, с увеличением номера моды выходят на насыщение.

\section{Финансирование работы}

Работа выполнена при поддержке грантов РФФИ № 14-01-00170 и № 14-08-00240.

\section{Конфликт интересов}

Авторы заявляют, что у них нет конфликта интересов. 


\section{Список литературы}

[1] Rayleigh (Strutt J.W.) // Phil. Mag. 1882. Vol. 14. P. 184-186.

[2] Doyle A., Moffet D.R., Vonnegut B. // J. Coll. Sci. 1964. Vol. 19. P. 136-143.

[3] Berg T.G.O., Trainor R.J., Vaughan U. // J. Atmosph. Sci. 1970. Vol. 27. N 11. P. 1173-1181.

[4] Schweizer J.D., Hanson D.N. // J. Coll. Int. Sci. 1971. Vol. 35. N 3. P. $417-423$.

[5] Roulleau M., Desbois M. // J. Atmosph. Sci. 1972. Vol. 29. N 4. P. 565-569.

[6] Duft D., Lebbeus H., Huber B.A. // Phys. Rev. Lett. 2002. Vol. 89. N 8. P. 1-4.

[7] Grimm R.L., Beauchamp J.L. // J. Phys. Chem. B. 2005. Vol. 109. P. 8244-8250.

[8] Fong Chee Sheng, Black N.D., Kiefer P.A., Shaw R.A. // Am. J. Phys. 2007. Vol. 75. N 6. P. 499-503.

[9] Hunter H.C., Ray Asit K. // Phys. Chem. Chemical Physics. 2009. Vol. 11. N 29. P. 6156-6165.

[10] Курчатов И.В., Наследов Д.Н., Семенов Н.Н., Харитон Ю.Б. Электронные явления. Л.: ОНТИ-ХИМТЕОРЕТ, 1935. $388 \mathrm{c}$.

[11] Григорьев А.И., Жаров А.Н., Ширяева С.О. // ЖТФ. 2005. T. 75. Вып. 8. С. 44-53.

[12] Brazier-Smith P.R. // J. Roy. Met. Soc. 1972. Vol. 98. P. 434-441.

[13] Ширяева С.О., Петрушов Н.А., Григорьев А.И. // ЖТФ. 2015. Т. 85. Вып. 8. С.33-39.

[14] Ширяева С.О., Петрушов Н.А., Григорьев А.И. // ЖТФ. 2016. Т. 86. Вып. 1. С.37-44.

[15] Френкель Я.И. // ЖЭТФ. 1936. Т. 6. № 4. С.348-350.

[16] Ландау Л.Д., Лифиии Е.М. Теоретическая физика. Т. 6. Гидродинамика. М.: Наука, 1986. 736 с.

[17] Ландау Л.Д., Лифиии Е.М. Теоретическая физика. Т. 2. Теория поля. М.: Наука, 1973. 504 с.

[18] Найфе А.X. Методы возмущений. М.: Мир, 1976. 455 с.

[19] Taylor G.I. // Proc. Roy. Soc. London. 1964. Vol. A280. P. 383-397.

[20] Hendrics C.D., Schneider J.M. // J. Amer. Phys. 1963. Vol. 1. N 6. P. $450-453$. 